\title{
Concurrent Dermatophytosis in Atopic Dogs
}

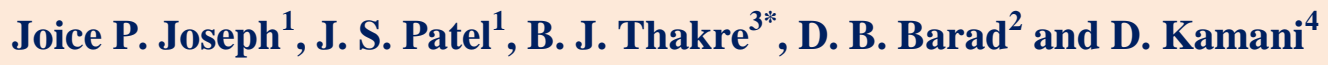 \\ ${ }^{1}$ Teaching Veterinary Clinical Complex, ${ }^{2}$ Department of Veterinary Parasitology, \\ ${ }^{3}$ Department of Veterinary Microbiology, ${ }^{4}$ Department of Veterinary Medicine, College of \\ Veterinary Science \& A. H., JAU, Junagadh - 362001 (Gujarat), India
}

*Corresponding author

\begin{tabular}{|c|c|}
\hline Keywords & A B S T R A C T \\
\hline $\begin{array}{l}\text { Dermatophytosis, } \\
\text { Atopic dermatitis }\end{array}$ & \multirow{3}{*}{$\begin{array}{l}\text { Twenty atopic dogs presented during the period of study were checked for } \\
\text { fungal infections using trichography, wood's lamp examination and fungal } \\
\text { culture. Four }(2 \%) \text { animals were found affected with dermatophyte } \\
\text { infections along with atopic dermatitis indicating the importance of } \\
\text { concurrent dermatophytosis in atopic dogs. }\end{array}$} \\
\hline Article Info & \\
\hline $\begin{array}{l}\text { Accepted: } \\
\text { 17 September } 2019 \\
\text { Available Online: } \\
10 \text { October } 2019\end{array}$ & \\
\hline
\end{tabular}

\section{Introduction}

Atopy is one of the common underlying skin diseases in dogs; it is a multifaceted disease associated with exposure to various offending agents such as environmental and food allergens (Favrot et al., 2010). Genetic mutations lead to alterations in the final keratinization of the epidermis and dysfunction of skin barrier facilitating the transepidermal penetration of environmental allergens, microbial antigens and primary irritants causing exacerbation of innate and adaptive immune responses (Hillier, 2002). This condition could be complicated with different secondary or concurrent skin infections. Identifying such infections is needed for successful treatment of atopic dermatitis.

\section{Materials and Methods}

Study was conducted at Teaching Veterinary Clinical Complex, Veterinary College, JAU, Junagadh. Twenty five atopic dogs (out of 221 dogs with dermatitis) were identified using intradermal test, Favrot's criteria and nonresponse to rational treatment during the period of study (one year). Diseased were subjected to different fungal detection tests including wood's lamp examination, trichography and fungal culture. Skin lesions were examined using wood's lamp kept 4 to 5 inches from lesion and lesions were tested in a dark room. Hairs from affected skin were plucked with forceps in the direction of the 
hair growth and were placed in a drop of mineral oil on a slide. It was then examined under low - power magnification $(10 \mathrm{x})$ after application of coverslip. Also skin scrapings from the lesions were collected under sterile precautions and were inoculated on dermatophyte test medium (DTM) added with dermatosupplement and sabouraud dextrose agar (SDA). Both were incubated at room temperature for 5 days. Morphology of microbial colony was studied for identification of fungus. Microscopical examination of fungal colonies using lactophenol cotton blue staining was also carried out for confirmation of diagnosis.

\section{Results and Discussion}

Four atopic dogs $(2 \%)$ were identified to suffer from concurrent dermatophyte infections. Bright apple green fluorescence was observed on Wood's lamp examination (Fig. 1). Microscopical examination revealed fungal spores attached to hairs (Fig. 2). Sabouraud dextrose agar and dermatophyte test medium agar showed white spreading colonies (Fig. 3 \& 4). Also colour of dermatophyte test medium agar changed from yellow to red by 5 days. Microscopical examination using lactophenol cotton blue revealed numerous microconidia of dermatophytes confirming dermatophyte infection in atopic dogs. All the affected animals were responding to treatment for atopy along with topical application of antifungal agent also.

Majority of secondary infections in atopic dogs are commensal organisms. But concurrent infections of dermatophytes can complicate this skin condition. Lockwood (2017) also reported concurrent infection of dermatophyte in an atopic dog with improper treatment. Wilson et al., (1993) and Klein et al., (1999) observed infections of dermatophytes during flaring up of atopic dermatitis. Fungi can act as either allergen or secondary infectious agent. So use of antifungal drug is important in atopic dogs with fungal infection. Also dogs with repeated occurrence of dermatitis along with dermatophytosis should also be checked for atopy.

Fig.1 Lesions in dermatophytosis observed through Wood's Lamp

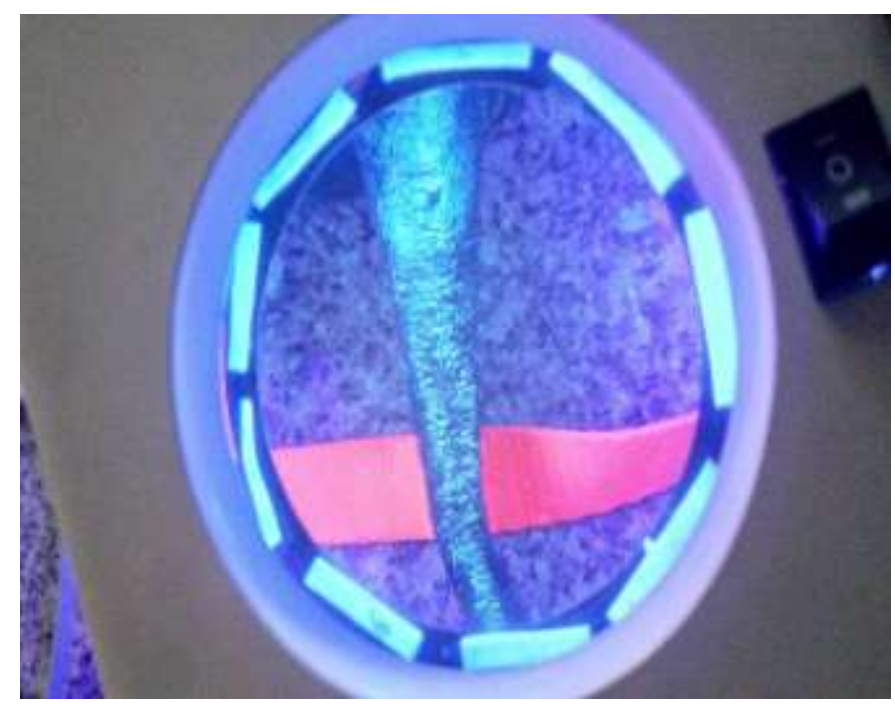


Fig.2 Ectothrix on hair in Trichography

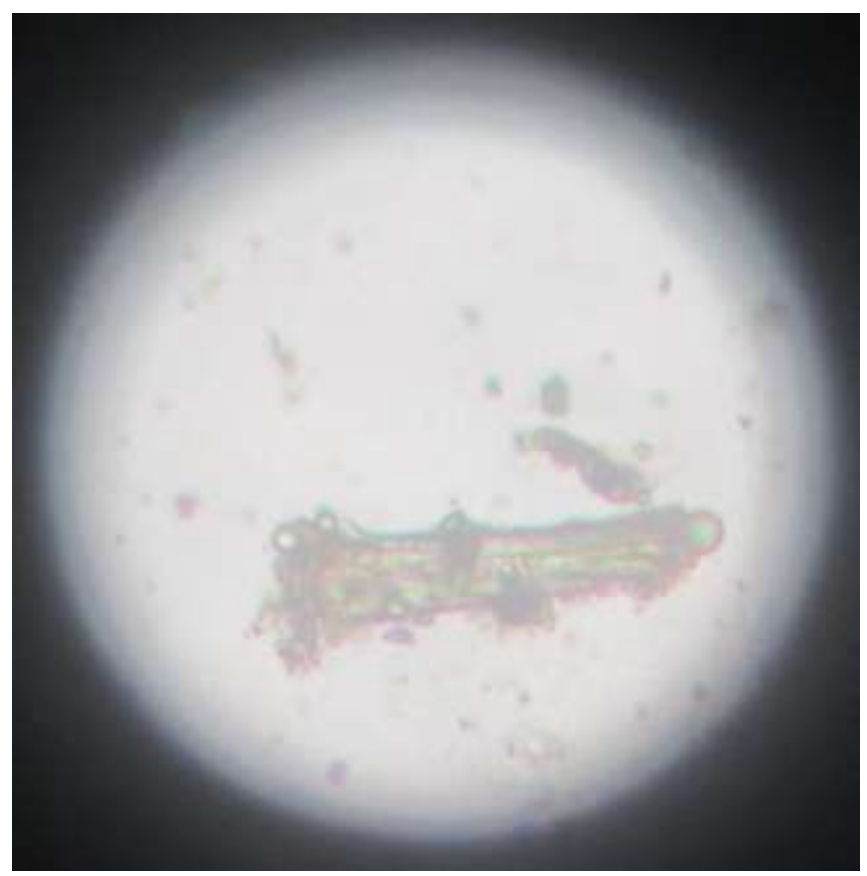

Fig.3 Dermatophyte cultures in SDA and DTM

SDA

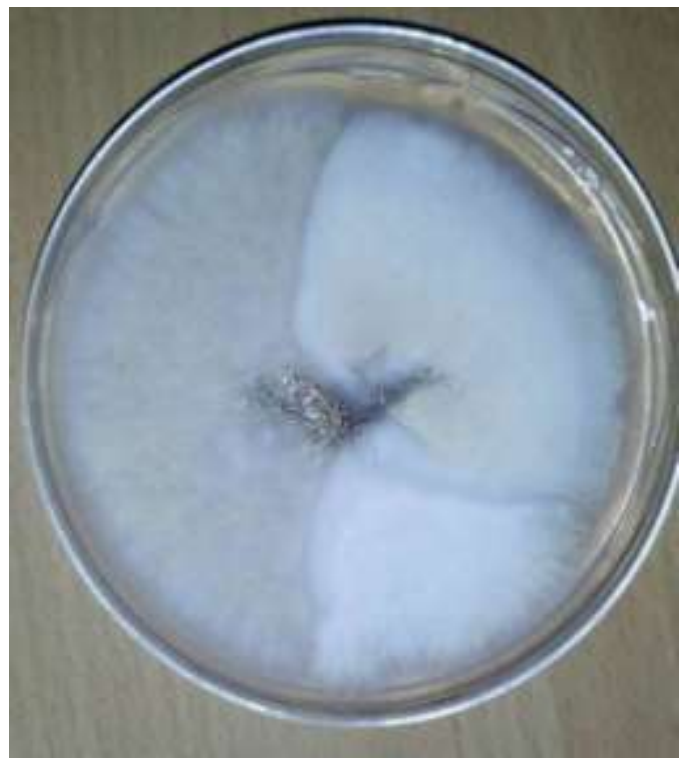

DTM

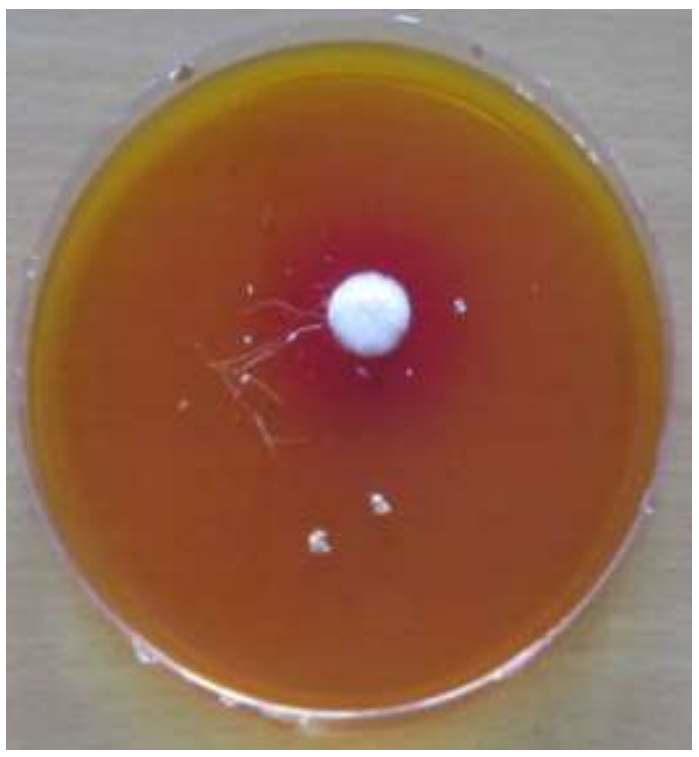


Fig.4 Microscopical examination of fungal culture

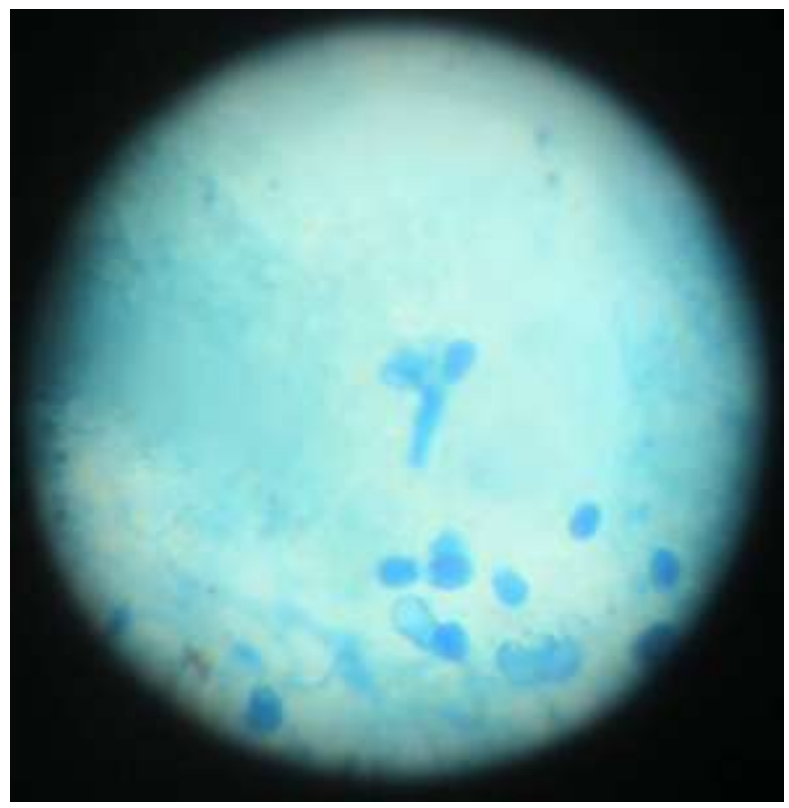

\section{References}

Favrot, C., Steffan, J., Seewald, W. and Picco, F. A. (2010). Prospective study on the clinical features of chronic canine atopic dermatitis and its diagnosis. Vet. Dermatol. 21(1): 23 - 31.

Hillier A. (2002). Definitively diagnosing atopic dermatitis in dogs. Vet. Med. 97(3): 198 - 208.

Klein, P. A., Clark, R. A. and Nicol, N. H. (1999). Acute infection with Trichophyton rubrum associated with flares of atopic dermatitis. Cutis. 63(3): $171-172$.
Lockwood, S. L., Mount, R., Lewis, T. P. and Schick, A. E. (2017). Concurrent development of generalised demodicosis, dermatophytosis and meticillin-resistant Staphylococcus pseudintermedius secondary to inappropriate treatment of atopic dermatitis in an adult dog. Vet. Rec. 5(1): 1 - 9 .

Wilson, B. B., Deuell, B. and Mills, T. A. (1993). Atopic dermatitis associated with dermatophyte infection and Trichophyton hypersensitivity. Cutis. 51(3): 191 - 192.

\section{How to cite this article:}

Joice P. Joseph, J. S. Patel, B. J. Thakre, D. B. Barad and Kamani, D. 2019. Concurrent Dermatophytosis in Atopic Dogs. Int.J.Curr.Microbiol.App.Sci. 8(10): 2484-2487. doi: https://doi.org/10.20546/ijcmas.2019.810.288 